\title{
A comparison of the prognostic value of BNP versus NT-proBNP after hospitalisation for heart failure
}

\author{
G. C. M. Linssen ${ }^{1,2} \cdot$ T. Jaarsma ${ }^{3} \cdot$ H. L. Hillege ${ }^{1,4} \cdot$ A. A. Voors ${ }^{1} \cdot$ D. J. van Veldhuisen ${ }^{1}$ \\ Published online: 7 August 2018 \\ (c) The Author(s) 2018
}

\begin{abstract}
Aims Concentrations of circulating B-type natriuretic peptides provide important prognostic information in heart failure (HF) patients. We directly compared the prognostic performance of brain natriuretic peptide (BNP) versus N-terminal-proBNP (NT-proBNP) measurements in a large population of HF patients at hospital discharge after an admission for decompensated HF.

Methods and results BNP and NT-proBNP were measured in 563 stable HF patients before discharge. All patients were followed for a fixed period of 18 months. The primary endpoint was time to first major event (HF hospitalisation or death). Patients were in NYHA class II (47\%) or III/IV (53\%) at discharge and the mean age of the patients was $71 \pm 11$ years, 217 (39\%) females, mean left ventricular ejection fraction was $0.32 \pm 0.14$ and $234(42 \%)$ had an ischaemic aetiology of HF. During the study, 236 patients (42\%) reached the primary endpoint. Multivariate odds ratios of the primary endpoint for doubling of baseline levels of BNP and NT-proBNP were 1.46 (95\% CI 1.19-1.80, $p<0.001$ ) and 1.45 (95\% CI 1.18-1.78, $p<0.001)$, respectively. The multivariable adjusted areas under the receiver-operating characteristic curve for prediction of the primary endpoint for doubling of BNP and NT-proBNP were 0.69 and 0.68 , respectively. Direct comparison of the prognostic value of BNP and NT-proBNP did not reveal significant differences.

Conclusions BNP and NT-proBNP at discharge for hospitalisation for HF are powerful, and equally strong and independent predictors of all-cause death and HF rehospitalisation.
\end{abstract}

Keywords Heart failure $\cdot$ Biomarkers $\cdot$ B-type natriuretic peptides $\cdot$ BNP $\cdot$ NT-proBNP $\cdot$ Prognosis

\section{What's new?}

- In patients with decompensated heart failure (HF), both B-type natriuretic peptides (BNP and NT-proBNP) are equally strong and independent predictors of outcome (HF rehospitalisation or death)

G. C. M. Linssen

g.linssen@zgt.nl

1 Department of Cardiology, University Medical Center Groningen, University of Groningen, Groningen, The Netherlands

2 Department of Cardiology, Hospital Group Twente, Almelo and Hengelo, The Netherlands

3 Department of Social and Welfare Studies, Faculty of Health Sciences, Linköping University, Norrköping, Sweden

4 Department of Epidemiology, University Medical Center Groningen, University of Groningen, Groningen, The Netherlands
- Both natriuretic peptides have comparable predictive accuracy at hospital discharge for HF.

- Clinically relevant for disease monitoring in HF patients treated with exogenous BNP or sacubitril/valsartan.

- Important for the selection of patients in trials and usage of natriuretic peptides as endpoints.

\section{Introduction}

Hospitalisation for acute heart failure (HF) syndromes portends a poor prognosis in patients with chronic HF [1]. Brain natriuretic peptides (BNP) and its equimolarly secreted amino-terminal fragment (NT-proBNP) are strong independent predictors of mortality and cardiovascular (CV) events in patients with heart failure (HF) [2-4]. Therefore, these biomarkers can help to identify patients at high risk for premature death or HF (re)hospitalisation.

Although both natriuretic peptides are frequently used, direct comparative studies on the prognostic value of BNP and NT-proBNP are scarce. Since BNP and NT-proBNP 
differ with regard to their biological activity and half-life, in vitro stability and clearance mechanisms, potential differences regarding their prognostic value may exist. In the ValHeFT study the prognostic values of BNP and NT-proBNP were compared in patients with stable chronic HF and in that study NT-proBNP was better in terms of predicting outcome [5]. However, predicting outcome after hospital discharge for worsening HF may be more difficult, but is clinically relevant. In this condition, only the findings from relatively small comparative studies are available [6-8].

Therefore, the purpose of our present study was to directly compare the prognostic performance of BNP versus NT-pro-BNP measurements in a large population of HF patients at hospital discharge after an admission for decompensated HF.

\section{Methods}

\section{Patient population and study design}

This analysis was performed as part of the Coordinating study evaluating Outcomes of Advising and Counselling in Heart Failure (COACH) study, a multicentre, randomised, open trial with blinded endpoint evaluation, in which 1,023 patients were enrolled. It was designed to compare basic support and intensive support in patients with chronic HF to a control group receiving 'usual' care, as described in detail before [9]. All patients had been admitted to hospital with symptoms of HF, New York Heart Association (NYHA) functional class II-IV. Patients were \pm 18 years of age and had evidence of structural underlying heart disease, as shown by cardiovascular imaging. Both patients with an impaired and those with a preserved left ventricular ejection fraction could participate. Before discharge from the hospital (i.e. before inclusion into the study), patients had to be stable on standard medication for $\mathrm{HF}$, at the discretion of the physician and if tolerated. After inclusion, all patients were followed for a fixed time period of 18 months. Primary endpoints were time to death or rehospitalisation for HF, and the number of days lost to death or hospitalisation. The Medical Ethics Committee approved the study protocol and all patients provided written informed consent. Institutional review board approval was given for all 17 participating Dutch centres. The primary results of the $\mathrm{COACH}$ study have been published previously [10].

In the current analyses, we only investigated $\mathrm{COACH}$ patients in whom measurements of plasma levels of both BNP and NT-proBNP at hospital discharge for decompensated chronic heart failure (baseline) were available. Of the 1,023 patients included in the $\mathrm{COACH}$ study, 563 patients had both BNP and NT-proBNP plasma levels available. The main reason for missing BNP and NT-proBNP data was temporary unavailability of the necessary laboratory facilities, usually in the starting phase of the study. Details have been published previously [11]. We studied the primary endpoint, time to first major event (HF hospitalisation or death).

Previously we reported specifically on the prognostic significance of BNP (and NT-proBNP) in heart failure patients with reduced and preserved left ventricular ejection fraction (LVEF) ( $\leq 40 \%$ and $>40 \%$, respectively) who participated in the $\mathrm{COACH}$ study [3]. In the current analyses we introduced LVEF as a continuous variable.

\section{Statistical analyses}

Continuous variables with a normal distribution are expressed as means with standard deviation (SD). Nominal variables are expressed as $n(\%)$. Levels of BNP and NTproBNP with a skewed distribution are given as medians with interquartile range (IQR). Differences in continuous variables were evaluated by Student's $t$-test or Mann-Whitney-U tests, depending on normality of data. Categorical clinical variables were compared with the Fisher's exact test or chi-square test. To achieve a constant variance, natriuretic peptide values were logarithmically transformed. BNP levels were correlated with NT-proBNP levels using Spearman's rank correlation coefficient.

The primary endpoint was time to first major event (HF hospitalisation or death). To estimate the size of the effect, odds ratios (ORs) with $95 \%$ confidence intervals (CIs) were calculated with the use of logistic regression models. A stepwise approach was used.

Risk estimates should be interpreted as the relative risk if values of BNP or NT-proBNP were doubled (e.g., from 10 to $20 \mathrm{pg} / \mathrm{ml}$ ). From logistic regression analysis the predictive values of BNP and NT-proBNP were determined and the area under the receiver operating characteristics (ROC) curves (AUC) for quantification of the predictive accuracy were calculated. An ROC area of 0.5 signifies no discriminatory value, while an area of 1.0 means perfect discrimination for prediction of those with and without an endpoint during follow-up.

Furthermore, a direct comparison of single levels in the regression models in $\mathrm{COACH}$ patients was part of this analysis.

All reported probability values are two-tailed and $p<0.05$ was considered statistically significant. Analyses were performed using STATA software (STATA version 10.0, College Station, Texas, USA). 
Table 1 Baseline characteristics of the study group at hospital discharge

\begin{tabular}{lc}
\hline & Study group $(n=563)$ \\
\hline Female gender & $217(39 \%)$ \\
Age (years) & $71 \pm 11$ \\
NYHA class III or IV & $294(53 \%)$ \\
LVEF & $0.32 \pm 0.14$ \\
Body mass index $\left(\mathrm{kg} / \mathrm{m}^{2}\right)$ & $26 \pm 5$ \\
Aetiology of heart failure & \\
- Ischaemic heart disease & $234(42 \%)$ \\
- Non-ischaemic heart disease & $329(58 \%)$ \\
Previously hospitalised for HF & $192(34 \%)$ \\
Comorbidities & \\
- Hypertension & $249(44 \%)$ \\
- Atrial fibrillation & $258(46 \%)$ \\
- Diabetes & $167(30 \%)$ \\
- Stroke & $51(9 \%)$ \\
- COPD & $156(28 \%)$ \\
Medication & \\
- ACE-I and/or ARB & $463(82 \%)$ \\
- $\beta$-blockers & $375(67 \%)$ \\
- Diuretics ${ }^{\text {b }}$ & $538(96 \%)$ \\
Laboratory values & \\
- Haemoglobin $(\mathrm{mmol} / \mathrm{l})$ & $8.2 \pm 1.3$ \\
- eGFR (ml/min/1.73 m $\left.{ }^{2}\right)$ & $54 \pm 20$ \\
- BNP (pg/ml ${ }^{\mathrm{c}}$ & $447(196-906)$ \\
- NT-proBNP $(\mathrm{pg} / \mathrm{ml})^{\mathrm{c}}$ & $2,528(1289-5615)$ \\
\hline
\end{tabular}

SI conversion factors: To convert NT-proBNP to picomoles per litre, divide by 8.46 ; BNP to picomoles per litre, divide by 3.47 ; haemoglobin to grams per litre, divide by 0.62

All continuous variables are presented as mean $\pm \mathrm{SD}$

$A C E-I$ angiotensin-converting enzyme inhibitor, $A R B$ angiotensin receptor blocker, $B N P$ brain natriuretic peptide, $C O P D$ chronic obstructive pulmonary disease, $e G F R$ estimated glomerular filtration rate, $H F$ heart failure, $L V E F$ left ventricular ejection fraction, NT-proBNP N-terminal prohormone B-type natriuretic peptide, NYHA New York Heart Association

${ }^{a}$ NYHA class at hospital discharge

bIncludes loop diuretics, thiazides, and aldosterone antagonists ${ }^{\mathrm{c}} \mathrm{A}$ continuous variable is presented as median value $\left(25^{\text {th }}-75^{\text {th }}\right.$ percentiles)

\section{Results}

\section{Baseline characteristics and outcome}

The study group at baseline comprised 563 HF patients: $217(39 \%)$ females, the mean age of the patients was $71 \pm 11$ years. Patients were in NYHA class II $(47 \%)$ or III/IV (53\%) at discharge. Both in the total cohort of $\mathrm{COACH}(n=1,023)$ and in our subgroup $(n=563)$, only $4 \%$ of HF patients were in NYHA class IV at hospital discharge. Therefore, we clustered NYHA class III and IV in the current analyses.
The mean LVEF was $0.32 \pm 0.14$ and $234(42 \%)$ had an ischaemic aetiology of HF. Mean estimated glomerular filtration rate (eGFR) was $54 \pm 20 \mathrm{ml} / \mathrm{min} / 1.73 \mathrm{~m}^{2}$. There were no significant differences in baseline characteristics between patients who participated in this study and in the main COACH cohort. In Tab. 1 baseline characteristics of the study group $(n=563)$ at hospital discharge are presented.

During the 18-month study, the combined endpoint occurred in 236 patients $(42 \%)$ of the whole population.

\section{BNP, NT-proBNP and outcome}

At hospital discharge after an admission for decompensated HF, median $\left(25^{\text {th }}-75^{\text {th }}\right.$ percentiles $)$ BNP and NT-proBNP levels in the 563 study patients were 447 (196-906) and $2,528(1,289-5,615) \mathrm{pg} / \mathrm{ml}$, respectively (Tab. 1$)$. We found



Fig. 1 Multivariable adjusted probability of outcome according to the BNP level at hospital discharge (on a log transformed scale)

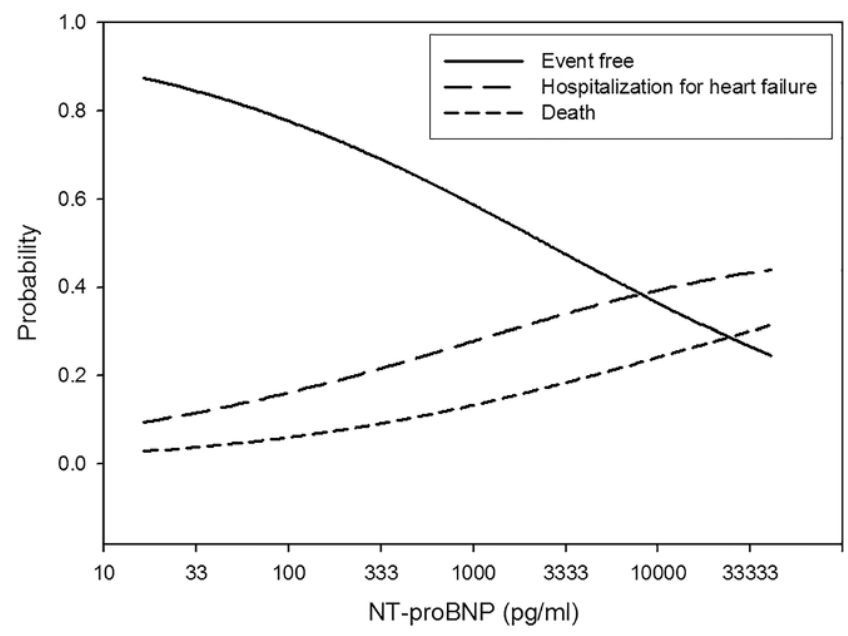

Fig. 2 Multivariable adjusted probability of outcome according to the NT-proBNP level at hospital discharge (on a log transformed scale) 
a strong association between BNP and NT-proBNP concentrations (correlation coefficients $0.82, p<0.001$ ). BNP at baseline was significantly $(p<0.05)$ related to: NYHA class, LVEF, body mass index (BMI), and HF underlying disease (ischaemic vs. non-ischaemic). NT-proBNP at baseline was significantly related to age, NYHA class, LVEF, BMI, HF underlying disease (ischaemic vs. non-ischaemic), haemoglobin and eGFR.

The baseline median BNP and NT-proBNP levels were higher in patients $(n=236,42 \%)$ who reached the primary endpoint compared with those $(n=337,58 \%)$ who remained free of this endpoint: 559 vs. $352 \mathrm{pg} / \mathrm{ml}, p<0.001$; and 3,396 vs. $2,011 \mathrm{pg} / \mathrm{ml}, p<0.001$, respectively.

The multivariable adjusted probability for event-free survival and for the separate endpoints (HF hospitalisation and all-cause death) according to the baseline BNP and NTproBNP levels, respectively, are depicted in Figs. 1 and 2.

\section{Prognostic value of a single predischarge measurement of BNP and NT-proBNP}

Unadjusted, age- and gender-adjusted and multivariate ORs of the primary endpoint for doubling of baseline levels of BNP and NT-proBNP are presented in Tables 2 and 3.

Multivariate ORs (adjusted for age, NYHA class, LVEF, BMI, HF underlying disease (ischaemic vs. non-ischaemic), and eGFR) were 1.46 (95\% CI $1.19-1.80, p<0.001)$ and 1.45 (95\% CI 1.18-1.78, $p<0.001)$, respectively for BNP and NT-proBNP. In addition, both BNP and NT-proBNP were independent predictors of the separate endpoints of HF hospitalisation and all-cause death.

Table 2 Odds ratios for outcome according to doubling of B-type natriuretic peptide (BNP) at hospital discharge for heart failure

\begin{tabular}{lllr}
\hline & Odds ratio & $95 \%$ CI & $P$ value \\
\hline For primary endpoint & & & \\
Unadjusted model & 1.30 & $1.16-1.45$ & $<0.001$ \\
Model 1 & 1.29 & $1.15-1.45$ & $<0.001$ \\
Model 2 & 1.46 & $1.19-1.80$ & $<0.001$ \\
For HF hospitalisation & & & \\
Unadjusted model & 1.24 & $1.09-1.41$ & $<0.001$ \\
Model 1 & 1.25 & $1.09-1.41$ & $<0.001$ \\
Model 2 & 1.42 & $1.20-1.68$ & $<0.001$ \\
For all-cause death & & & \\
Unadjusted model & 1.41 & $1.20-1.66$ & $<0.001$ \\
Model 1 & 1.40 & $1.19-1.66$ & $<0.001$ \\
Model 2 & 1.37 & $1.12-1.69$ & 0.003 \\
\hline
\end{tabular}

Model 1: Age- and gender-adjusted model

Model 2: Multivariate model adjusted for significant covariates: age, NYHA class, LVEF, BMI, HF underlying disease (ischaemic vs. non-ischaemic), and eGFR
Table 3 Odds ratios for outcome according to doubling of N-terminal pro-B-type natriuretic peptide (NT-proBNP) at hospital discharge for heart failure

\begin{tabular}{llll}
\hline & Odds ratio & $95 \%$ CI & $P$ value \\
\hline For primary endpoint & & & \\
Unadjusted model & 1.34 & $1.20-1.49$ & $<0.001$ \\
Model 1 & 1.31 & $1.17-1.46$ & $<0.001$ \\
Model 2 & 1.45 & $1.18-1.78$ & $<0.001$ \\
For HF hospitalisation & & & \\
Unadjusted model & 1.23 & $1.10-1.40$ & $<0.001$ \\
Model 1 & 1.22 & $1.08-1.38$ & $<0.001$ \\
Model 2 & 1.33 & $1.13-1.56$ & $<0.001$ \\
For all-cause death & & & \\
Unadjusted model & 1.55 & $1.33-1.82$ & $<0.001$ \\
Model 1 & 1.50 & $1.28-1.76$ & $<0.001$ \\
Model 2 & 1.45 & $1.17-1.78$ & $<0.001$ \\
\hline
\end{tabular}

Model 1: Age and gender adjusted model

Model 2: Multivariate model adjusted for significant covariates: age, NYHA class, LVEF, BMI, HF underlying disease (ischaemic vs. non-ischaemic), and eGFR

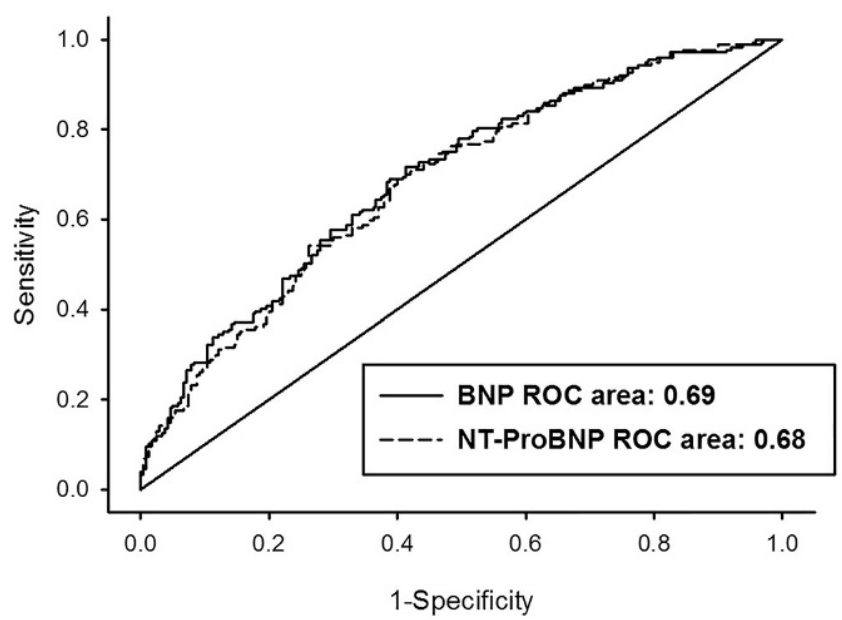

Fig. 3 Receiver operating characteristics (ROC) curves (multivariable adjusted) of discharge levels of BNP and NT-proBNP levels in predicting hospitalisation for $\mathrm{HF}$ or death, both at 18 months

\section{Predictive accuracy of BNP versus NT-proBNP}

The areas under the ROC curve for prediction of the primary endpoint, calculated for each doubling of BNP and NTproBNP, were 0.69 and 0.68 respectively.

The corresponding, multivariable adjusted ROC curves for each doubling of BNP and NT-proBNP are plotted in Fig. 3. There were no significant differences in AUC between BNP and NT-proBNP. 


\section{Discussion}

The main finding of the present study is that BNP and NTproBNP are equally strong and independent predictors of outcome at hospital discharge. Direct comparison of the predictive accuracy of BNP and NT-proBNP did not reveal significant differences.

Rational use of these peptides is currently recommended in patients with HF in several clinical settings: on admission for decompensated heart failure, after a major treatment effect, at hospital discharge when euvolaemia is reached, and during ambulatory follow-up [2, 12]. A single measurement of a B-type natriuretic peptide provides strong and independent prognostic information in patients with heart failure.

Previously, we observed in the COACH study that BNP levels are lower in patients with $\mathrm{HF}$ with preserved ejection fraction $(\mathrm{HFpEF})$ than in patients with $\mathrm{HF}$ with reduced ejection fraction (HFrEF), but for a given BNP level, the prognosis in patients with HFpEF is as poor as in those with HFrEF [3]. Patients with HFpEF were older, more often female, and had a higher systolic blood pressure and body mass index, and haemoglobin levels were lower than in those with HFrEF.

Inclusion of BNP or NT-proBNP concentrations in the diagnostic algorithm of $\mathrm{HF}$ is important both in clinical decision making and for proper design of trials. In concordance with proposed pathophysiological concepts, our recent analyses of multiple biomarkers from $\mathrm{COACH}$ showed that in HFpEF patients, inflammation and angiogenesis-mediated interactions were predominant, while stretch-mediated interactions were found in $\mathrm{HFrEF}[2,13-16]$.

Interestingly, in HFpEF patients with low BNP $(<100 \mathrm{pg} / \mathrm{ml})$ or low NT-proBNP $(<300 \mathrm{pg} / \mathrm{ml})$, quality of life, heart failure-related symptoms and clinical outcomes were similar to those with elevated BNP levels [17].

Furthermore, we reported on the added value of a diverse group of 29 biomarkers on top of a clinical risk model in $\mathrm{COACH}$ with and without NT-proBNP. Low risk was defined as a biomarker cut-off at the 10th percentile associated with high positive predictive value for 30-day and 180-day mortality and HF rehospitalisation [18].

HF symptom relief and euvolaemia reached at hospital discharge is an important point of time for measurement of BNP or NT-proBNP. Their concentrations may serve as targets for optimal fluid status or markers of disease evolution during follow-up in addition to clinical parameters, and in biomarker-guided management in HFrEF [19].

\section{BNP versus NT-proBNP in heart failure}

Plasma levels of the biological active BNP and inactive N-terminal fragment of BNP are closely correlated with each other in HF patients, as confirmed by the results of our study. The Valsartan Heart Failure Trial (Val-HeFT) study group provided a direct comparison of the prognostic value of BNP and NT-proBNP in 3,916 patients with chronic and stable HF [5]. They found that both peptides were powerful independent markers of outcome in HF, but NT-proBNP was superior to BNP in predicting mortality and morbidity or hospitalisation for HF. In 164 patients (99\% men) hospitalised for decompensated HF, Waldo and coworkers found that admission and discharge NT-proBNP (AUC 0.788 and AUC 0.834) had superior prognostic power for all-cause mortality within 90 days post-discharge, when compared with BNP (AUC 0.644, $p<0.01$ and AUC 0.709, $p<0.01$ ) [6]. Also in a small study, 171 patients with acute HF, Noveanu and coworkers reported that predischarge levels of BNP and NT-proBNP reliably predicted one-year mortality (AUC 0.78 and 0.77 respectively); however, prediction of one-year HF readmission was poor for both markers [7]. In a subgroup analysis of 306 patients with acute HF (FINNAKVA cohort), both BNP and NT-proBNP failed to improve prediction of 5-year survival [8]. In 563 patients, we found that the prognostic performance (all-cause mortality or HF hospitalisation during 18 months) of BNP and NTproBNP at the time of hospital discharge were comparable.

From other studies, it became apparent that patient- and assay-related factors influence both BNP and NT-proBNP concentrations $[2,20]$. In head-to-head comparisons, distinct discrepancies in individual patients demonstrated that both markers are clinically not completely equivalent [7-9, 21, 22]. Furthermore, BNP was found to be more sensitive to rapid haemodynamic changes in acute heart failure than NT-proBNP $[8,23]$. Importantly, monitoring by means of BNP testing of chronic heart failure patients on exogenous administered BNP or guideline-recommended sacubitril-valsartan treatment, may be impaired, in contrast to NT-proBNP, which is not a substrate for neprilysin inhibition [24]. So, these issues should be taken into account while applying serial testing for risk stratification and (long-term) monitoring of HF patients. Analyses of NT-proBNP and BNP in HFrEF patients who participated in the PARADIGM-HF study revealed that NT-proBNP decreases on treatment with sacubutril-valsartan, reflecting reduced cardiac wall stress, while BNP increases, reflecting drug action [25]. However, the relative increases in BNP concentrations in that randomised study were small (median baseline BNP value approximately $200 \mathrm{pg} / \mathrm{ml}$ to a median of about $225 \mathrm{pg} / \mathrm{ml}$ in the sacubitril-valsartan arm at 8-month follow-up).

Furthermore, it is unlikely that small increments of BNP on that drug will interfere with the diagnostic applications of BNP in patients with acute or decompensated heart failure, usually associated with large increments of BNP. 


\section{Study limitations}

The present analysis was observational in design and is therefore only hypothesis-generating. In the current retrospective analysis of a randomised controlled trial, we only included medical therapy at hospital discharge. In that way, modifications in the drug treatment and non-pharmacological therapy during follow-up were not accounted for in our analysis. The $\mathrm{COACH}$ study was powered for the primary composite endpoint, time to hospitalisation for HF or allcause mortality, but not for the separate, secondary endpoint all-cause mortality.

Also, the $\mathrm{COACH}$ study was conducted at a time when not all currently recommended drugs for chronic HF were used and use of device therapy has also markedly increased since then, which may have affected our findings $[12,26]$.

\section{Conclusions}

Plasma concentrations of both BNP and NT-proBNP at discharge after hospitalisation for HF are equally strong and independent predictors of all-cause death and HF rehospitalisation.

Funding The $\mathrm{COACH}$ study was supported by a large program grant from the Netherlands Heart Foundation (grant 2000Z003). Additional unrestricted grants were obtained from Biosite Europe, France (Brain Natriuretic Peptide [BNP]), Roche Diagnostics, the Netherlands (Nterminal-proBNP), and Novartis Pharma BV, the Netherlands.

Conflict of interest G.C.M. Linssen, T. Jaarsma, H.L. Hillege, A.A. Voors and D.J. van Veldhuisen declare that they have no competing interests.

Open Access This article is distributed under the terms of the Creative Commons Attribution 4.0 International License (http:// creativecommons.org/licenses/by/4.0/), which permits unrestricted use, distribution, and reproduction in any medium, provided you give appropriate credit to the original author(s) and the source, provide a link to the Creative Commons license, and indicate if changes were made.

\section{References}

1. Metra M, Teerlink JR. Heart failure. Lancet. 2017;390:1981-95.

2. Chow SL, Maisel AS, Anand I, et al. Role of biomarkers for the prevention, assessment, and management of heart failure: A scientific statement from the American Heart Association. Circulation. 2017;135:e1054-e91.

3. van Veldhuisen DJ, Linssen GCM, Jaarsma T, et al. B-type natriuretic peptide and prognosis in heart failure patients with preserved and reduced ejection fraction. J Am Coll Cardiol. 2013;61:1498-506.

4. Zile MR, Claggett BL, Prescott MF, et al. Prognostic implications of changes in N-terminal pro-B-type natriuretic peptide in patients with heart failure. J Am Coll Cardiol. 2016;68:2425-36.

5. Masson S, Latini R, Anand IS, et al. Direct comparison of B-type natriuretic peptide (BNP) and amino-terminal proBNP in a large population of patients with chronic and symptomatic heart fail- ure: the Valsartan Heart Failure (Val-HeFT) data. Clin Chem. 2006;52:1528-38.

6. Waldo SW, Beede J, Isakson S, et al. Pro-B-type natriuretic peptide levels in acute decompensated heart failure. J Am Coll Cardiol. 2008;51:1874-82.

7. Noveanu M, Breidthardt T, Potocki M, et al. Direct comparison of serial B-type natriuretic peptide and NT-proBNP levels for prediction of short- and long-term outcome in acute decompensated heart failure. Crit Care. 2011;15:R1.

8. Seronde MF, Gayat E, Logeart D, et al. Comparison of the diagnostic and prognostic values of B-type and atrial-type natriuretic peptides in acute heart failure. Int J Cardiol. 2013;168:3404-11.

9. Jaarsma T, van der Wal MH, Hogenhuis J, et al. Design and methodology of the $\mathrm{COACH}$ study: a multicenter randomised Coordinating study evaluating Outcomes of Advising and Counselling in Heart failure. Eur J Heart Fail. 2004;6:227-33.

10. Jaarsma T, van der Wal MH, Lesman-Leegte I, et al. Effect of moderate or intensive disease management program on outcome in patients with heart failure: Coordinating Study Evaluating Outcomes of Advising and Counseling in Heart Failure $(\mathrm{COACH})$. Arch Intern Med. 2008;168:316-24.

11. Hogenhuis J, Voors AA, Jaarsma T, et al. Anaemia and renal dysfunction are independently associated with BNP and NTproBNP levels in patients with heart failure. Eur J Heart Fail. 2007;9:787-94.

12. Ponikowski P, Voors AA, Anker SD, et al. ESC Guidelines for the diagnosis and treatment of acute and chronic heart failure. Eur J Heart Fail. 2016;2016(18):891-975.

13. Anand IS, Rector TS, Cleland JG, et al. Prognostic value of baseline plasma amino-terminal pro-brain natriuretic peptide and its interactions with irbesartan treatment effects in patients with heart failure and preserved ejection fraction: findings from the I-PRESERVE trial. Circ Heart Fail. 2011;40:569-77.

14. Linssen GCM, Rienstra M, Jaarsma T, et al. Clinical and prognostic effects of atrial fibrillation in heart failure patients with reduced and preserved left ventricular ejection fraction. Eur J Heart Fail. 2011;13:1111-20.

15. Paulus WJ, Tschöpe C. A novel paradigm for heart failure with preserved ejection fraction: comorbidities drive myocardial dysfunction and remodeling through coronary microvascular endothelial inflammation. J Am Coll Cardiol. 2013;62:263-71.

16. Tromp J, Khan MA, Klip IT, et al. Biomarker profiles in heart failure patients with preserved and reduced ejection fraction. J Am Heart Assoc. 2017;6. pii: e003989. https://doi.org/10.1161/JAHA. 116.003989 .

17. Meijers WC, Hoekstra T, Jaarsma T, van Veldhuisen DJ, de Boer RA. Patients with heart failure with preserved ejection fraction and low levels of natriuretic peptides. Neth Heart J. 2016;24:287-95.

18. Meijers WC, de Boer RA, van Veldhuisen DJ, et al. Biomarkers and low risk in heart failure. Data from COACH and TRIUMPH. Eur J Heart Fail. 2015;17:1271-82.

19. Brunner-La Rocca HP, Eurlings L, Richards AM, et al. Which heart failure patients profit from natriuretic peptide guided therapy? A meta-analysis from individual patient data of randomized trials. Eur J Heart Fail. 2015;17:1252-61.

20. Hogenhuis J, Voors AA, Jaarsma T, Hillege HL, Boomsma F, van Veldhuisen DJ. Influence of age on natriuretic peptides in patients with chronic heart failure: a comparison between ANP/NT-ANP and BNP/NT-proBNP. Eur J Heart Fail. 2005;7:81-6.

21. Vanderheyden M, Bartunek J, Claeys G, Manoharan G, Beckers JF, Ide L. Head to head comparison of N-terminal pro-Btype natriuretic peptide and B-type natriuretic peptide in patients with/without left ventricular systolic dysfunction. Clin Biochem. 2006;39:640-5. 
22. Mair J, Gerda F, Renate H, Ulmer H, Andrea G, Pachinger O. Headto-head comparison of B-type natriuretic peptide (BNP) and NTproBNP in daily clinical practice. Int J Cardiol. 2008;124:244-6.

23. Mair J, Falkensammer G, Poelzl G, Hammerer-Lercher A, Griesmacher A, Pachinger O. B-type natriuretic peptide (BNP) is more sensitive to rapid hemodynamic changes in acute heart failure than N-terminal proBNP. Clin Chim Acta. 2007;379:163-6.

24. Mair J, Lindahl B, Giannitsis E, et al. Will sacubitril-valsartan diminish the clinical utility of B-type natriuretic peptide test- ing in acute cardiac care? Eur Heart J Acute Cardiovasc Care. 2017;6:321-8.

25. Packer M, McMurray JJ, Desai AS, et al. PARADIGM-HF Investigators and Coordinators. Angiotensin receptor neprilysin inhibition compared with enalapril on the risk of clinical progression in surviving patients with heart failure. Circulation. 2015;131:54-61.

26. van Veldhuisen DJ, Maass AH, Priori SG, et al. Implementation of device therapy (cardiac resynchronization therapy and implantable cardioverter defibrillator) for patients with heart failure in Europe: changes from 2004 to 2008. Eur J Heart Fail. 2009;11:1143-51.

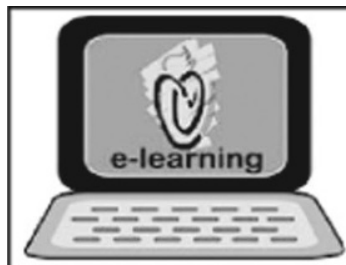

\section{CVOI E-learning formula!}

This is the CVOI e-learning article. The author has prepared 10 questions which are available through the website of the Cardiovascular Educational Institute (CVOI). Please follow the instructions below.

After finishing the questions you will be asked to fill in your name, hospital and e-mail address; then press the button 'verzenden'.

When 6 out of the 10 questions are answered correctly, you acquire 1 accreditation point granted by the Quality Committee of the Netherlands Society of Cardiology (NVVC). The acquired point will be credited to your personal file in the GAIA system. You will also receive an e-mail with all the correct answers.

Over a period of one year 10 e-learning articles will appear in 10 subsequent $\mathrm{NHJ}$ editions. In each edition the e-learning article will be recognisable by a special icon. On an annual basis you can collect 10 accreditation points. The accreditation points are credited in the GAIA system by the CVOI.

If you need additional information, please contact the CVOI by e-mail: cvoi@cvoi.org or by phone: 030-2345001.

J.J. Piek

Chief editor NHJ
K.B. Schick

Coordinator CVOI 